\title{
Classification of intrahepatic biliary strictures and assessment of outcome in living donor liver transplantation
}

\author{
Hansang Park' ${ }^{1}$ Namjoon Yi ${ }^{1}$, Euisoo Han ${ }^{1}$, Kyung-Suk Suh${ }^{1}$, Joonkoo Han², Kwang-Woong Lee ${ }^{1}$, YoungRok Choi ${ }^{1}$, \\ Sukkyun Hong ${ }^{1}$, Saejin Park ${ }^{2}$ \\ ${ }^{1}$ Department of Surgery, Seoul National University Hospital, Seoul, Korea
${ }^{2}$ Department of Radiology, Seoul National University Hospital, Seoul, Korea
}

Background: Biliary complications account for unsolved common complications after living donor liver transplantation (LDLT). However, intrahepatic biliary stricture (IHBS) after LDLT is not common but requires intensive care. The purpose of this study is to classify IHBS and to evaluate the prognosis of IHBS after LDLT.

Methods: From 2011 to 2018, 868 cases of the right liver LDLT were enrolled. According to cholangiographic appearance, types of biliary stricture were classified into four, based on level and number of involved branches: type 1 (anastomosis or the 1st order branch; single), type 2 (the 2nd order branch; a. single, b. double, c. extended to the 3rd order branch), type 3 (multifocal), type 4 (diffuse necrosis). IHBS was defined as type 2, 3 and 4 . We evaluated biliary intervention free period more than 1 year after last intervention (IFY), intervention frequency per year and clinical relapse after IFY.

Results: The overall incidence of biliary stricture including IHBS was $23 \%(n=198)$; IHBS was $9 \%(n=76)$. The most common type of stricture was type $1(n=122,62 \%)$ followed by type $2(n=66,33 \%), 3(n=6,3 \%)$ and $4(n=4,2 \%)$. Incidence of type 2 sub-classification consisted of $2 a(n=8,4 \%), 2 b(n=15,8 \%), 2 c(n=43,22 \%)$. IFY was more common in type $1(85 \%)$ and $2(a, 88 \% ; b, 87 \% ; c$, $72 \%)$ than type $3(67 \%)$ and $4(25 \%)(P<0.05)$. Intervention frequency per year was higher in type $4(12)$ than others (type 1,3 ; type $2 a, 2$; type $2 b, 4$; type $2 c, 5$; type 3,7$)(P<0.05)$. Clinical relapse after IFY was more common in type $4(50 \%)$ and $3(67 \%)$ than type $2(33 \%)$ and $1(37 \%)$ but it was not significantly different $(P>0.05)$.

Conclusions: IHBS was not rare in right liver LDLT. Although multifocal stricture or diffuse necrosis of intrahepatic bile duct were uncommon, they required more interventions.

Corresponding author: Namjoon Yi

E-mail: gsleenj@hanmail.net

(c) The Korean Society for Transplantation

This is an Open Access article distributed under the terms of the Creative Commons Attribution Non-Commercial License (http://creativecommons.org/licenses/by-nc/4.0/) which permits unrestricted non-commercial use, distribution, and reproduction in any medium, provided the original work is properly cited. 